JOURNAL OF

SYNCHROTRON

RADIATION

ISSN 1600-5775

\section{Characterizing transmissive diamond gratings as beam splitters for the hard $X$-ray single-shot spectrometer of the European $\mathrm{XFEL}^{1}$}

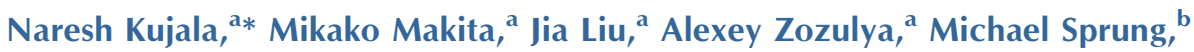 \\ Christian David $^{\mathrm{c}}$ and Jan Grünert ${ }^{\mathrm{a}}$
}

Received 21 December 2018

Accepted 9 March 2019

Edited by D. Cocco, SLAC National Accelerator Laboratory, USA

\footnotetext{
${ }^{1}$ This article will form part of a virtual special issue containing papers presented at the PhotonDiag2018 workshop.
}

Keywords: CVD diamond; free-standing diamond membrane; X-ray free-electron laser; photon diagnostics; diffraction efficiency; grating structures; HIREX diagnostic spectrometer.

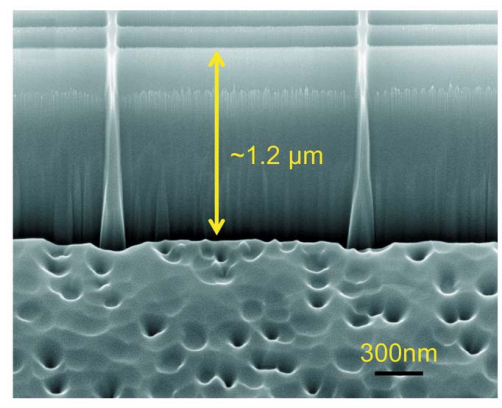

C 2019 International Union of Crystallography

\author{
${ }^{\text {a} E}$ uropean XFEL GmbH, Holzkoppel 4, 22869 Schenefeld, Germany, ${ }^{\mathbf{b}}$ P10 Beamline, PETRA III, Deutsches Elektronen- \\ Synchrotron (DESY), Notkestrasse 85, 22607 Hamburg, Germany, and ${ }^{\mathbf{c}}$ Paul Scherrer Institut (PSI), 5232 Villigen, \\ Switzerland. *Correspondence e-mail: naresh.kujala@xfel.eu
}

The European X-ray Free Electron Laser (EuXFEL) offers intense, coherent femtosecond pulses, resulting in characteristic peak brilliance values a billion times higher than that of conventional synchrotron facilities. Such pulses result in extreme peak radiation levels of the order of terawatts $\mathrm{cm}^{-2}$ for any optical component in the beam and can exceed the ablation threshold of many materials. Diamond is considered the optimal material for such applications due to its high thermal conductivity $\left(2052 \mathrm{~W} \mathrm{mK}^{-1}\right.$ at $\left.300 \mathrm{~K}\right)$ and low absorption for hard X-rays. Grating structures were fabricated on free-standing CVD diamond of $10 \mu \mathrm{m}$ thickness with $500 \mu \mathrm{m}$ silicon substrate support. The grating structures were produced by electron-beam lithography at the Laboratory for Microand Nanotechnology, Paul Scherrer Institut, Switzerland. The grating lines were etched to a depth of $1.2 \mu \mathrm{m}$, resulting in an aspect ratio of 16 . The characterization measurements with X-rays were performed on transmissive diamond gratings of $150 \mathrm{~nm}$ pitch at the P10 beamline of PETRA III, DESY. In this paper, the gratings are briefly described, and a measured diffraction efficiency of $0.75 \%$ at $6 \mathrm{keV}$ in the first-order diffraction is shown; the variation of the diffraction efficiency across the grating surface is presented.

\section{Introduction}

Over the past decade, several X-ray free-electron laser (XFEL) facilities have been developed and constructed around the world. Two technologies have been pursued to construct electron accelerators for XFEL applications: warm, normal-conducting machines and cold, super-conducting accelerators (Emma et al., 2010; Allaria et al., 2012; Schreiber et al., 2012; Ishikawa et al., 2012; Harmand et al., 2013; Pellegrini et al., 2015). The European XFEL (EuXFEL) in the Hamburg metropolitan area, Germany, is an X-ray source based on a super-conducting linear accelerator which started user operation in fall 2017 (Saldin et al., 2000; Altarelli et al., 2006; Tschentscher et al., 2017; Decking et al., 2019). EuXFEL operates with a $10 \mathrm{~Hz}$ bunch train structure. Each train can contain up to 2700 pulses. Individual X-ray pulses are of the order of 2 fs to 100 fs in duration, contain energy of the order of several $\mathrm{mJ}$, and are separated by $222 \mathrm{~ns}$ (at the nominal intra-train repetition rate of $4.5 \mathrm{MHz}$ ). This unique time structure provides not only very high peak brilliance but also the highest average brilliance over all of the other XFEL facilities. There are in total three undulators that can produce self-amplified spontaneous emission (SASE) radiation, among which two hard X-ray undulators, SASE1 and SASE2, generate hard XFEL radiation up to $25 \mathrm{keV}$ while the third undulator, SASE3, provides soft X-rays up to $3 \mathrm{keV}$. 
One important problem in the use of the XFEL beam is the so-called single-shot damage. A large number of photons arrive within less than $100 \mathrm{fs}$, therefore thermal transport processes cannot remove substantial heat during the pulse, even for excellent heat conductors such as copper or diamond. For focused beam conditions (typically smaller than $50 \mu \mathrm{m}$ diameter), most materials will vaporize on an ultrafast time scale due to the single XFEL pulse absorption within the $\mathrm{X}$-ray penetration depth. More resistant materials are those with low atomic number where the absorption per atom is low. Most components that are directly exposed to XFEL radiation are therefore made of boron carbide or diamond, e.g. slits, beam stops, shutters, collimators and attenuators. The average power during a train of X-ray pulses can be as high as several $\mathrm{kW}$ depending on the energy per pulse and the number of pulses within the train. Diamond is considered as the most optimal material for such applications due to its high thermal conductivity $\left(2052 \mathrm{~W} \mathrm{mK}^{-1}\right.$ at $\left.300 \mathrm{~K}\right)$ and low absorption for hard X-rays.

The stochastic nature of SASE FEL radiation gives rise to shot-to-shot fluctuations for most beam properties, including pulse energy, spatial profile, wavefront, pulse duration, temporal profile and spectrum. The X-ray photon diagnostics group (XPD) at the European XFEL provides essential information to the machine for setup, optimization and operation of the accelerator, undulator and X-ray optics (Grünert, 2012; Grünert et al., 2015, 2019). In particular, the resulting spectrum will vary considerably from shot to shot. In order to monitor these variations, the high-resolution hard X-ray single-shot spectrometer (HIREX diagnostic spectrometer) was installed in the photon tunnel XTD9 of the SASE1 undulator beamline (Kujala et al., 2019). HIREX is an on-line device, and it contains a linear transmissive diamond diffraction grating to split off a small fraction of the photon beam, a bent Si crystal as a dispersive element, and a MHz-repetitionrate strip detector. In this paper, we report on the fabrication of gratings, measurements of the diffraction efficiency in the various diffraction orders, and efficiency maps over the grating surface, obtained at the P10 beamline of PETRA III at DESY, Hamburg, Germany.

\section{Grating fabrication}

Free-standing polycrystalline diamond membranes made by chemical vapour deposition (CVD) were used to fabricate the grating structures. Diamond membranes supported by a silicon frame were purchased from Diamond Materials $\mathrm{GmbH}$, Germany. The grating structures were fabricated at the Laboratory for Micro- and Nanotechnology at the Paul Scherrer Institut (PSI), Switzerland, using electron-beam lithography (EBL) and inductively coupled plasma (ICP) etching in an oxygen atmosphere. Figs. $1(a)$ and $1(b)$ show a sketch and an image of such a grating. The membrane has a diameter of $5 \mathrm{~mm}$ and a thickness of $10 \mu \mathrm{m}$ and is supported by a $500 \mu \mathrm{m}$-thick silicon frame of size $10 \mathrm{~mm} \times 10 \mathrm{~mm}$. The grating lines are produced over a large area of $2 \mathrm{~mm} \times 2 \mathrm{~mm}$, large enough to make alignment convenient and simple, and to
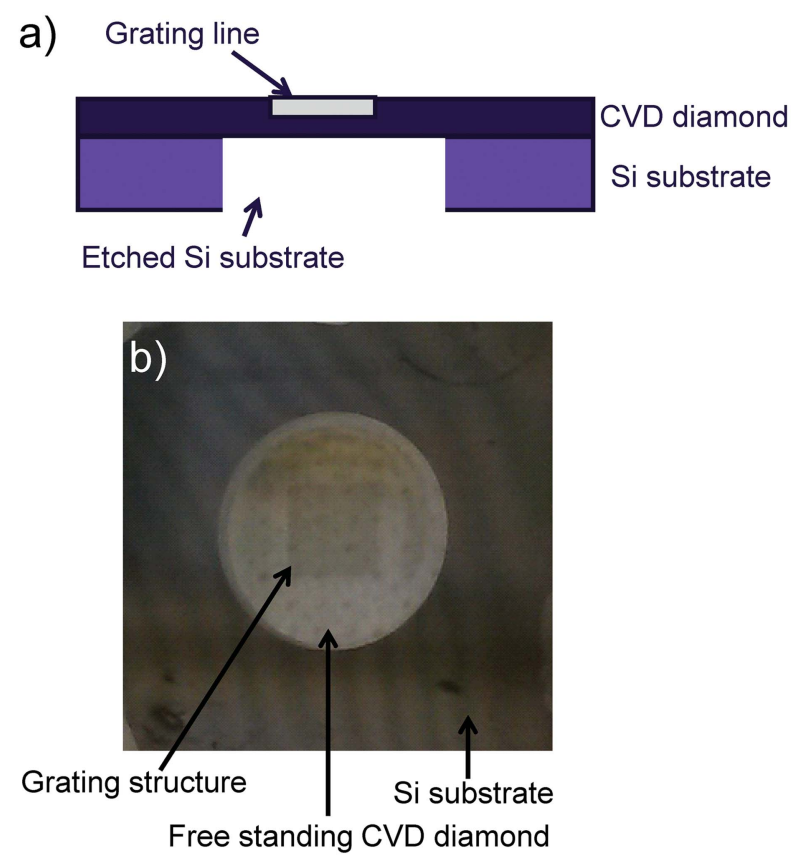

Figure 1

(a) Sketch of the grating design etched into a free-standing diamond membrane. (b) Image of a grating with grating lines extending over $2 \mathrm{~mm} \times 2 \mathrm{~mm}$ on the Si substrate.

accommodate the beam size of the European XFEL photon energy. To avoid collapse of the grating lines during resist development and ICP etching, the grating lines were connected by support structures. The support structures are a set of periodic lines perpendicular to the grating lines, having a pitch that is ten times larger than the grating lines. The diffracted intensity from the support structures is several orders of magnitude smaller than that from the grating lines. Further details about the gratings' fabrication are given elsewhere (David et al., 2011; Karvinen et al., 2012; Makita et al., 2015, 2017). A measurement of the grating efficiency, along with its spatial distribution across the grating area, is essential as feedback for the optimization of the fabrication process. Fig. 2(a) shows a scanning electron microscope (SEM) image of grating lines of size $2 \mathrm{~mm} \times 2 \mathrm{~mm}$ and pitch $150 \mathrm{~nm}$. Fig. 2(b) shows an SEM image of gratings taken at a $45^{\circ}$ tilted angle and etched to a depth of $1.2 \mu \mathrm{m}$, resulting in an aspect ratio of 16. There is no remaining resist in the SEM image from the fabrication and it was removed before experimental measurements were performed.

\section{Experimental setup}

Several linear transmissive gratings with different pitches were fabricated for hard X-rays in the energy range 5-20 keV for the HIREX diagnostic spectrometer. To understand the diffraction efficiencies and the structural homogeneities of the gratings we have performed an experiment at beamline P10 of PETRA III (Kalbfleisch et al., 2011). We have tested a grating with a pitch of $150 \mathrm{~nm}$ at three different photon energies, $6 \mathrm{keV}, 8 \mathrm{keV}$ and $12 \mathrm{keV}$. Fig. 3 shows a sketch of the beamline 

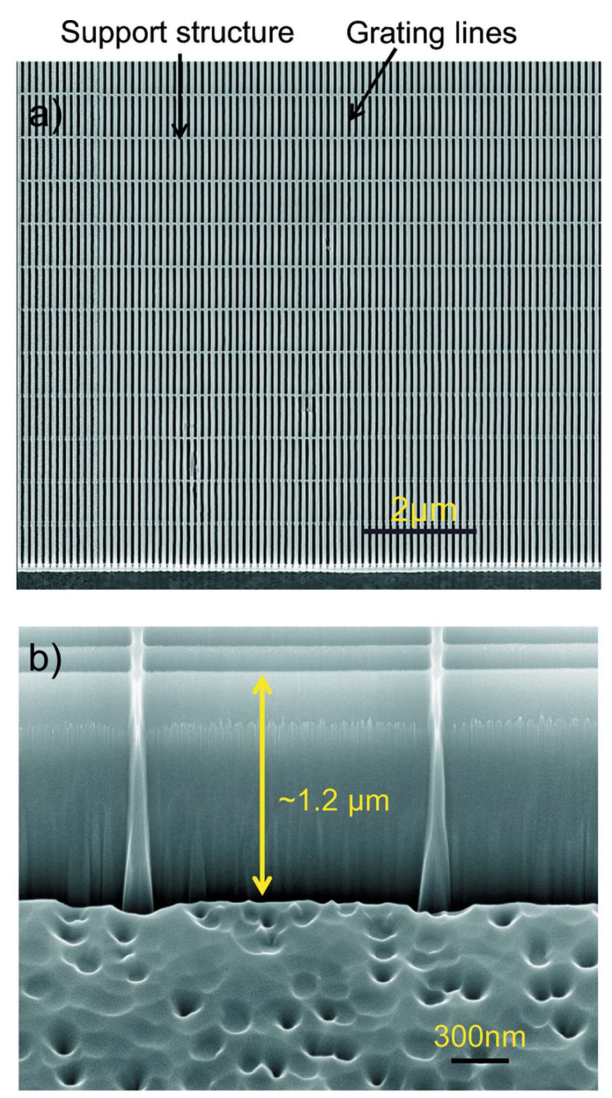

Figure 2

(a) Top view of an SEM image of the linear grating with a pitch of $150 \mathrm{~nm}$ and support structure for stability of the grating lines. (b) Side view SEM image taken at a $45^{\circ}$ titled angle of the linear grating with a pitch of $150 \mathrm{~nm}$ and a depth of $1.2 \mu \mathrm{m}$.

and Fig. 4 shows a photograph of the Coherent Diffraction Imaging (CDI) setup in the experimental hutch EH2 at the P10 beamline. The beamline has a silicon (111) double-crystal monochromator (DCM). The beam size is defined by slits which are located downstream of the DCM. The gratings were mounted on a holder which itself was mounted on a DN100 flange and placed in the sample chamber. For the measurements an unfocused beam of area $100 \mu \mathrm{m} \times 100 \mu \mathrm{m}$ was adjusted by the slits. The 2D Lambda detector developed at DESY was used for data collection of diffraction orders from

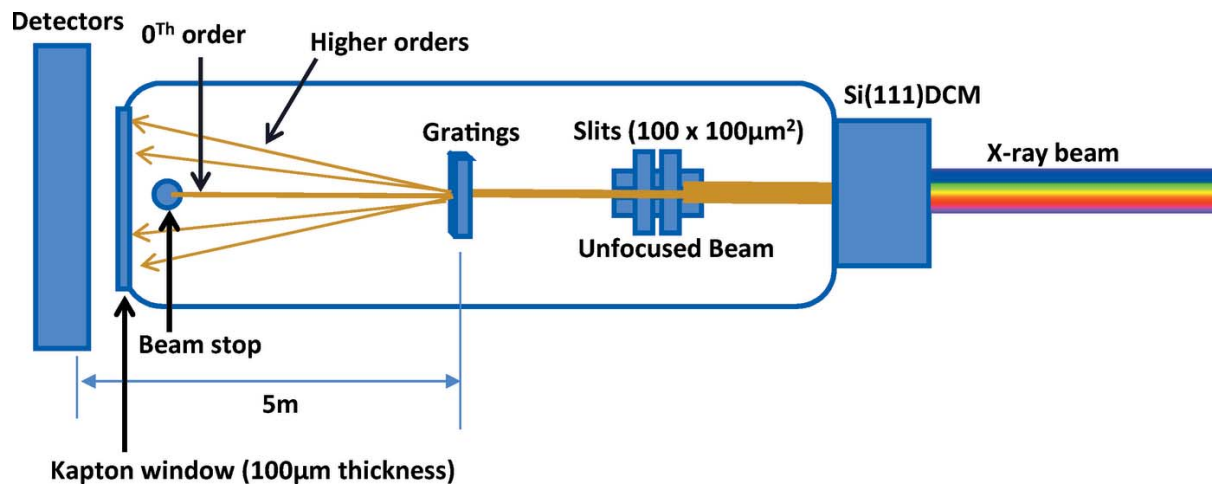

Figure 3

Sketch of the P10 beamline for measuring the grating diffraction efficiencies.

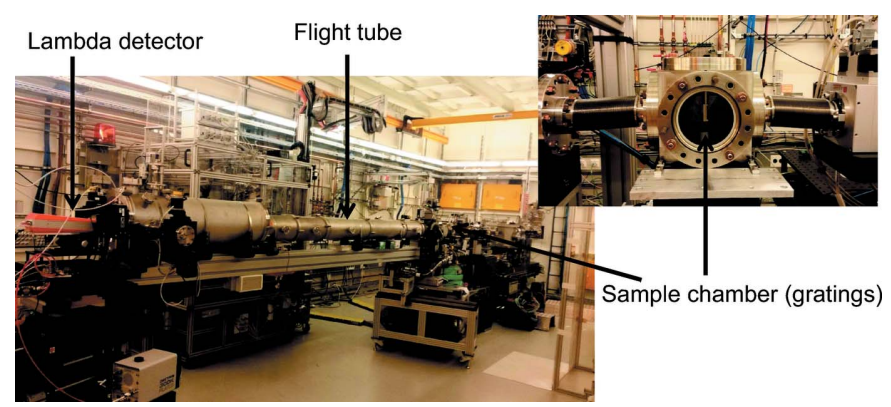

Figure 4

Photographs of the experimental hutch 2 of beamline P10 and the sample vacuum chamber (with installed gratings), flight tube and Lambda detector.

the gratings. The Lambda detector is based on the Medipix 3 chipset and has $55 \mu \mathrm{m} \times 55 \mu \mathrm{m}$ pixel size and an area of 1536 pixels $\times 512$ pixels $(84.5 \mathrm{~mm} \times 28.2 \mathrm{~mm})$ and a frame rate up to $2 \mathrm{kHz}$. The data were acquired with an exposure time of $100 \mathrm{~ms}$. A beam stop was placed in front of the detector to block the direct beam. The detector was placed outside the vacuum system. A flight tube with a $100 \mu \mathrm{m}$-thick Kapton window at the exit flange provided an evacuated beam path between sample chamber and detectors. The zerothorder and first-order diffraction efficiencies were measured using a photodiode. In front of the photodiode a circular aperture of diameter $100 \mu \mathrm{m}$ was mounted on a linear stage, allowing measurement of the intensity from the diffraction order by scanning its position. The 2D Lambda detector was used to acquire the higher diffraction orders from the gratings. In order to achieve good separation between the zeroth order and higher orders, the detectors were placed $5 \mathrm{~m}$ downstream of the gratings.

\section{Results}

The diffraction efficiencies and the structural homogeneities of the gratings are of crucial importance to better understand and further optimize the fabrication process. Knowledge of these parameters is also crucial to model the grating performance and correctly adjust the absolute photon intensities in the diffraction orders for the beam splitter applications at the diagnostic spectrometer. The diffraction efficiency and homogeneity of the grating surfaces with pitches of $150 \mathrm{~nm}$ were tested for three different photon energies, $6 \mathrm{keV}, 8 \mathrm{keV}$ and $12 \mathrm{keV}$. The linear transmissive CVD diamond grating was placed normal to the incident beam and $2 \%$ of the beam was absorbed by the diamond membrane of the gratings at $6 \mathrm{keV}$. The grating lines were oriented in the vertical direction, so that the diffracted beams were deflected in the horizontal plane. The gratings were placed in a vacuum chamber with a vacuum level of the 

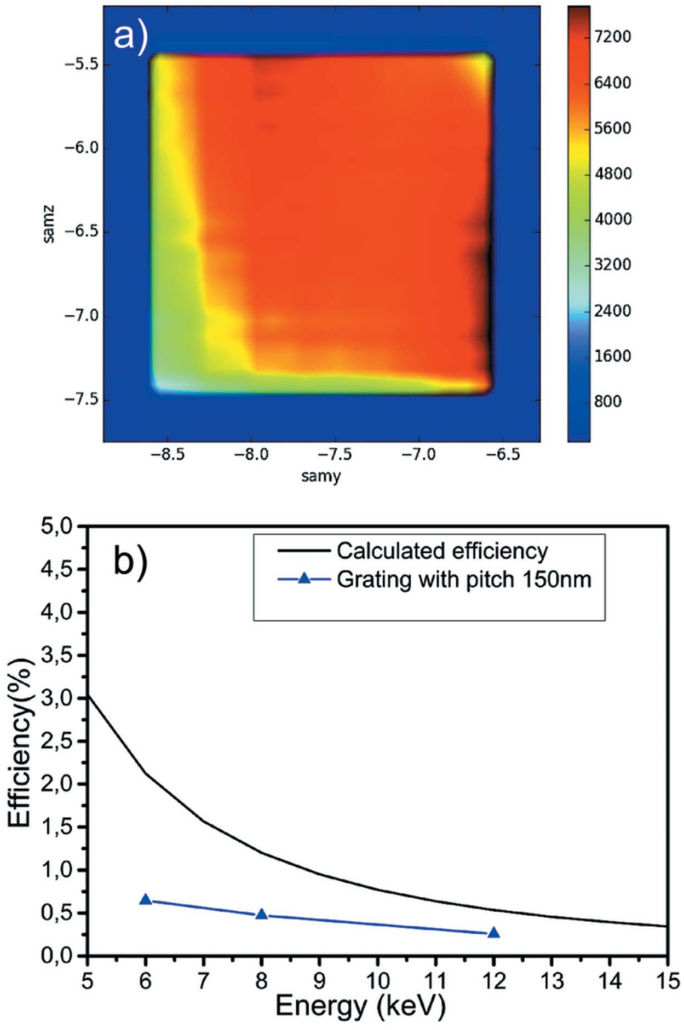

Figure 5

(a) Efficiency map of the first-order diffraction from gratings with $150 \mathrm{~nm}$ pitch at a photon energy of $6 \mathrm{keV}$. (b) Diffraction efficiency as a function of photon energy. The calculated values are shown as a black line. Measured diffraction efficiencies are shown as blue triangles.

order of $10^{-7}$ mbar; at the detector position the separations between the zeroth-order and first-order diffracted beam was $6.8 \mathrm{~mm}$ at $6 \mathrm{keV}$ and $3.3 \mathrm{~mm}$ at $12 \mathrm{keV}$ photon energy. The separation of orders with respect to the direct beam (zeroth order) can be calculated when the pitch of the gratings, the photon energy and the distance between gratings and detector are known. In order to investigate the homogeneity of the grating structures we have scanned the grating surface by moving the sample holder over an area of $2 \mathrm{~mm} \times 2 \mathrm{~mm}$ and acquired data with the photodiode. Fig. 5(a) shows the efficiency mesh in the first-order diffraction at a photon energy of $6 \mathrm{keV}$. The efficiency variation across most of the grating surface is within $20 \%$. However, the left bottom edge of the gratings has a $55 \%$ lower efficiency than measured at the centre of the surface, as shown in Fig. 5(a). This is due to the fact that the diamond membranes are not flat but exhibit a curvature, which results in errors in the focus settings during ebeam lithography. Errors in the focus setting have an effect on the electron-beam size and writing fields stitching accuracy. This then causes local variations of the grating line width and depth, or the duty cycle. Fig. 5(b) shows the measured and calculated diffraction efficiency as a function of photon energy for a grating with $150 \mathrm{~nm}$ pitch. The calculations for diffraction efficiency used publicly available data from the Berkeley Laboratory Centre for X-ray optics (http://henke.lbl.gov/). The diffraction efficiency was measured by the photodiode. The measured diffraction efficiency is $37 \%$ of the calculated values. This discrepancy in efficiency values between measurement and calculation arises from the fact that in theoretical calculations the structures consist of perfect binary squares and the fabrication imperfections are not taken into account. Fig. 6(a) shows the higher-order diffractions. The zeroth-order beam is blocked by a beamstop to protect the Lambda detector and to see the low-intensity signal coming from the diffraction orders. Data were collected for a photon energy of $6 \mathrm{keV}$. The diffraction spots are spread horizontally and are visible up to the fifth order of diffraction. The finerspaced diffraction spots along the vertical direction are caused by the support structures of the gratings, which are typically $80 \%$ less in intensity than those coming from the corresponding first-order diffraction spot. In between the diffraction orders there is another very weak intensity signal which is due to the intrinsic scattering function of the grating. Fig. 6(b) shows the intensity of the higher diffraction orders obtained from line profiles through the Lambda detector image. The negative-order diffraction intensity is slightly larger than that on the positive-order side which is mainly due to the fact that the gratings are not perfectly aligned to the incoming X-ray beam. We aligned the gratings perpendicular to the incident
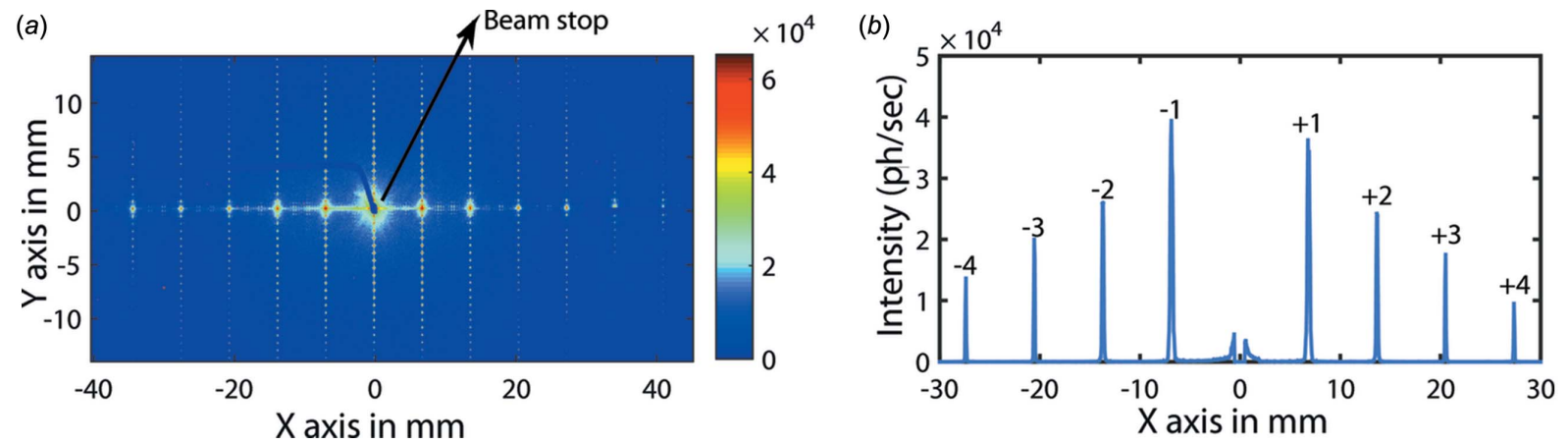

Figure 6

(a) Lambda detector spatial data showing the grating diffraction at a distance of $5 \mathrm{~m}$ downstream of the grating. The zeroth-order beam is blocked by a beam stop. The higher-order diffractions are spread in the horizontal direction. The vertical diffraction spots are due to support structures in the gratings. Those spots are very low in intensity with respect to the horizontal diffraction orders. $(b)$ Intensity line profile from the Lambda detector image across the grating diffraction orders up to the fourth order. 
$\mathrm{X}$-ray beam. However, we suspect there is slight misalignment with respect to the incident angle around the vertical axis which would explain the fact that the negative orders are slightly larger than the positive-order diffraction.

\section{Summary}

Linear transmissive CVD diamond gratings were tested as beam splitters at the P10 beamline at PETRA III, DESY. The results show that fabricated gratings with $150 \mathrm{~nm}$ pitch have homogeneous grating structures. The homogeneity of the grating structure measurement will be used as feedback information to further optimize the e-beam lithography parameters for grating fabrication. In particular, we can use the measurements to adjust the proper focusing of the electron beam across the curved diamond membrane surface. The measurement parameters are also used as feedback for operation of the gratings in the HIREX spectrometer. The diffraction efficiencies into first-order diffraction are sufficient to use these gratings as beam splitters. Non-invasive spectral measurements of the XFEL beam and the zeroth-order beam will be delivered to the scientific endstations for experiments. The efficiency variation map helps us in aligning the gratings to the beam to a position with homogeneous grating structure. The gratings are mounted on the linear manipulator which allows them to be aligned relative to the incoming X-rays such that impinge on the uniform grating structure which is obtained from the measurement results at the P10 beamline. The tested gratings are now installed in the HIREX diagnostic spectrometer. In the HIREX spectrometer we have installed $150 \mathrm{~nm}$ and $200 \mathrm{~nm}$ pitch gratings. The $200 \mathrm{~nm}$ pitch grating shows comparable results with the $150 \mathrm{~nm}$ grating. Depending on the photon energy, we plan to use the different grating pitches: the $200 \mathrm{~nm}$ pitch gratings will be used for the photon energy range $3-5 \mathrm{keV}$, while the $150 \mathrm{~nm}$ pitch gratings will be used for 5-20 keV. This diagnostic spectrometer is installed in the photon tunnel XTD9 of the SASE1 undulator beamline. The HIREX spectrometer is an online device using diamond diffraction gratings of different pitches as beam splitters. A silicon crystal with fixed bending radius is used as the energydispersive element. A Gotthard detector (Mozzanica et al., 2011) with $\mathrm{MHz}$ rate acquisition capability is used for recording spectra at high pulse repetition rates, whereas a Photonic Science sCMOS camera is used at low repetition rates. The characterization of diamond gratings using the highresolution small-angle X-ray scattering setup at P10 beamline provides an efficient approach for non-invasive studies of such optics and feedback for manufacturing of high-quality X-ray optics.

\section{Acknowledgements}

NK would like to thank P. Karvinen from Finnlitho Ltd for discussions about the project, Jens Rehanek from PSI Switzerland for discussions during the initial phase of the project, and Eckhard Wörner from Diamond Materials $\mathrm{GmbH}$, Freiburg, Germany, for providing the free-standing diamond membrane. All the authors would like to thank the P10 beamline staff for their support during the beam time. We also gratefully acknowledge the European XFEL management board members for supporting and funding this project.

\section{References}

Allaria, E., Appio, R., Badano, L., Barletta, W., Bassanese, S., Biedron, S. G., Borga, A., Busetto, E., Castronovo, D., Cinquegrana, P., Cleva, S., Cocco, D., Cornacchia, M., Craievich, P., Cudin, I., D'Auria, G., Dal Forno, M., Danailov, M. B., De Monte, R., De Ninno, G., Delgiusto, P., Demidovich, A., Di Mitri, S., Diviacco, B., Fabris, A., Fabris, R., Fawley, W., Ferianis, M., Ferrari, E., Ferry, S., Froehlich, L., Furlan, P., Gaio, G., Gelmetti, F., Giannessi, L., Giannini, M., Gobessi, R., Ivanov, R., Karantzoulis, E., Lonza, M., Lutman, A., Mahieu, B., Milloch, M., Milton, S. V., Musardo, M., Nikolov, I., Noe, S., Parmigiani, F., Penco, G., Petronio, M., Pivetta, L., Predonzani, M., Rossi, F., Rumiz, L., Salom, A., Scafuri, C., Serpico, C., Sigalotti, P., Spampinati, S., Spezzani, C., Svandrlik, M., Svetina, C., Tazzari, S., Trovo, M., Umer, R., Vascotto, A., Veronese, M., Visintini, R., Zaccaria, M., Zangrando, D. \& Zangrando, M. (2012). Nat. Photon. 6, 699-704.

Altarelli, M. et al. (2006). XFEL: The European X-Ray Free-Electron Laser - Technical Design Report. DESY 2006-097. DESY, Hamburg, Germany (doi:10.3204/DESY_06-097).

David, C., Gorelick, S., Rutishauser, S., Krzywinski, J., VilaComamala, J., Guzenko, V. A., Bunk, O., Färm, E., Ritala, M., Cammarata, M., Fritz, M. D., Barrett, R., Samoylova, L., Grünert, J. \& Sinn, H. (2011). Sci. Rep. 1, 57.

Decking, W. et al. (2019). Nat. Photon. Submitted.

Emma, P., Akre, R., Arthur, J., Bionta, R., Bostedt, C., Bozek, J., Brachmann, A., Bucksbaum, P., Coffee, R., Decker, F., Ding, Y., Dowell, D., Edstrom, S., Fisher, A., Frisch, J., Gilevich, S., Hastings, J., Hays, G., Hering, P., Huang, Z., Iverson, R., Loos, H. M., Messerschmidt, M., Miahnahri, A., Moeller, S., Nuhn, H., Pile, G., Ratner, D., Rzepiela, J., Schultz, D., Smith, T., Stefan, P., Tompkins, H., Turner, J., Welch, J., White, W., Wu, J., Yocky, G. \& Galayda, J. (2010). Nat. Photon. 4, 641-647.

Grünert, J. (2012). European XFEL Technical Report XFEL.EU TR2012-003. European XFEL, Schenefeld, Germany (doi:10.3204/ XFEL.EU/TR-2012-003).

Grünert, J., Carbonell, M. P., Dietrich, F., Falk, T., Freund, W., Koch, A., Kujala, N., Laksman, J., Laksman, J., Tiedtke, K., Jastrow, U. F., Sorokin, A., Syresin, E., Grebentsov, A. \& Brovko, O. (2019). J. Synchrotron Rad. 26. To be published.

Grünert, J., Koch, A., Kujala, N., Freund, W., Planas, M., Dietrich, F., Buck, J., Liu, J., Sinn, H., Dommach, M. \& Molodtsov, S. (2015). Proceedings of the 37th International Free Electron Laser Conference (FEL 2015), 23-28 August 2015, Daejeon, South Korea. WED03.

Harmand, M., Coffee, R., Bionta, M. R., Chollet, M., French, D., Zhu, D., Fritz, D. M., Lemke, H. T., Medvedev, N., Ziaja, B., Toleikis, S. \& Cammarata, M. (2013). Nat. Photon. 7, 215-218.

Ishikawa, T., Aoyagi, H., Asaka, T., Asano, Y., Azumi, N., Bizen, T., Ego, H., Fukami, K., Fukui, T., Furukawa, Y., Goto, S., Hanaki, H., Hara, T., Hasegawa, T., Hatsui, T., Higashiya, A., Hirono, T., Hosoda, N., Ishii, M., Inagaki, T., Inubushi, Y., Itoga, T., Joti, Y., Kago, M., Kameshima, T., Kimura, H., Kirihara, Y., Kiyomichi, A., Kobayashi, T., Kondo, C., Kudo, T., Maesaka, H., Maréchal, X. M., Masuda, T., Matsubara, S., Matsumoto, T., Matsushita, T., Matsui, S., Nagasono, M., Nariyama, N., Ohashi, H., Ohata, T., Ohshima, T., Ono, S., Otake, Y., Saji, C., Sakurai, T., Sato, T., Sawada, K., Seike, T., Shirasawa, K., Sugimoto, T., Suzuki, S., Takahashi, S., Takebe, H., Takeshita, K., Tamasaku, K., Tanaka, H., Tanaka, R., Tanaka, T., Togashi, T., Togawa, K., Tokuhisa, A., Tomizawa, H., Tono, K., Wu, S., Yabashi, M., Yamaga, M., Yamashita, A., Yanagida, K., 
Zhang, C., Shintake, T., Kitamura, H. \& Kumagai, N. (2012). Nat. Photon. 6, 540-544.

Kalbfleisch, S., Neubauer, H., Krüger, S. P., Bartels, M., Osterhoff, M., Mai, D. D., Giewekemeyer, K., Hartmann, B., Sprung, M., Salditt, T., McNulty, I., Eyberger, C. \& Lai, B. (2011). AIP Conf. Proc. 1365, 96-99.

Karvinen, P., Rutishauser, S., Mozzanica, A., Greiffenberg, D., Juranić, N. P., Menzel, A., Lutman, A., Krzywinski, J., Fritz, D. M., Lemke, H. T., Cammarata, M. \& David, C. (2012). Opt. Lett. 37, 5073.

Kujala, N., Freund, W., Koch, A., Planas, M., Liu, J., Dietrich, F. \& Grünert, J. (2019). In preparation.

Makita, M., Karvinen, P., Guzenko, V. A., Kujala, N., Vagovic, P. \& David, C. (2017). Microelectron. Eng. 176, 75-78.

Makita, M., Karvinen, P., Zhu, D., Juranic, P., Grünert, J., Cartier, S., Jungmann-Smit, J. H., Lemke, H. T., Mozzanica, A., Nelson, S.,
Patthey, L., Sikorski, M., Song, S., Feng, Y. \& David, C. (2015). Optica, 2, 912-916.

Mozzanica, A., Bergamaschi, A., Dinapoli, R., Graafsma, H., Henrich, B., Kraft, P., Johnson, I., Lohmann, M., Schmitt, B. \& Shi, X. (2011). Nucl. Instrum. Methods Phys. Res. A, 633, S29-S32. Pellegrini, C., Marinelli, A. \& Reiche, S. (2015). Rev. Mod. Phys. 88, 015006.

Saldin, E. L., Schneidmiller, E. V. \& Yurkov, M. V. (2000). The Physics of Free Electron Lasers, 1st ed. Berlin: Springer-Verlag.

Schreiber, S., Faatz, B., Feldhaus, J., Honkavaara, K., Treusch, R. \& Vogt, M. (2012). Proceedings of the 34th International Free-Electron Laser Conference (FEL2012), 26-31 August 2012, Nara, Japan, p. 37. MOPD01.

Tschentscher, T., Bressler, C., Grünert, J., Madsen, A., Mancuso, A. P., Meyer, M., Scherz, A., Sinn, H. \& Zastrau, U. (2017). Appl. Sci. 2017, 7, 592. 